\title{
On-surface synthesis of variable bandgap nanoporous graphene
}

Dingguan Wang, Xuefeng Lu, Arramel, Ming Yang, * Jishan Wu, *Andrew. T. S. Wee*

Dr. D. Wang, Dr. Arramel, Dr. M. Yang, Prof. A. T. S. Wee

Department of Physics, National University of Singapore, 2 Science Drive 3, Singapore

117551, Singapore

E-mail: phyweets@nus.edu.sg (A.T.S.W.)

Dr. D. Wang, Dr. X. Lu, Prof. J. Wu

Department of Chemistry, National University of Singapore, 3 Science Drive 3, Singapore

117543, Singapore

E-mail: chmwuj@nus.edu.sg (J.W.)

Dr. M. Yang

Department of Applied Physics, The Hong Kong Polytechnic University, Hung Hom,

Kowloon, Hong Kong, China

E-mail: mingyang@polyu.edu.hk (M.Y.)

Dr. X. Lu

Department of Materials Science, Fudan University, Shanghai 200438, China

Keywords: nanoporous graphene, on-surface synthesis, bandgap modulation, LT-STM/ncAFM, DFT calculation

Tuning the bandgap of nanoporous graphene is desirable for applications such as the charge transport layer in organic-hybrid devices. The holy grail in the field is the ability to synthesize 2D nanoporous graphene with variable pore sizes, and hence tuneable band gaps. Herein, we demonstrate the on-surface synthesis of nanoporous graphene with variable bandgaps. Two types of nanoporous graphene were synthesized via hierarchical C-C coupling, and verified by low-temperature scanning tunneling microscopy and non-contact atomic force microscopy with CO-terminated tip. Nanoporous graphene-1 is non-planar, and nanoporous graphene-2 is a single-atom thick planar sheet. Scanning tunneling spectroscopy measurements reveal that nanoporous graphene-2 has a bandgap of $3.8 \mathrm{eV}$, while nanoporous graphene-1 has a larger bandgap of $5.0 \mathrm{eV}$. Corroborated by first-principles calculations, we propose that the large bandgap opening is governed by the confinement of $\pi$-electrons induced by pore generation or the non-planar structure, and can be explained by Clar sextet theory. Our finding shows that 
by introducing nanopores, semimetallic graphene is converted into semiconducting nanoporous graphene-2 or insulating wide-bandgap nanoporous graphene-1.

\section{Introduction}

Graphene, a single-atom-thick carbon sheet, has gained widespread attention due to its unique structural and physical properties. ${ }^{[1]}$ Graphene shows extremely high mobility of electrons and holes, high mechanical strength, high thermal conductivity, high impermeability to any gases, good mechanical stability, and the large specific surface area. All these superlative merits make graphene promising for numerous applications. However, to date graphene has not found widespread applications in the electronic industry, even though the challenge of largesize fabrication of high-quality graphene has been largely overcome. ${ }^{[2]}$ The main issue for electronic applications is the lack of an intrinsic quasiparticle bandgap in graphene, which results in a very low on/off switching ratio in graphene field-effect transistors and other electronic devices. ${ }^{[3]}$

Many research strategies have addressed opening a bandgap in graphene, such as by physical/chemical doping, electric field application, 1D confinement, defect or strain engineering. ${ }^{[2]}$ For example, the bandgap opening in graphene can be realized by applying a large external strain to break its sub-lattice symmetry. ${ }^{[4]} 1 \mathrm{D}$ electron confinement is also another way to open a bandgap in graphene by cutting the $2 \mathrm{D}$ carbon sheet into $1 \mathrm{D}$ graphene nanoribbons. ${ }^{[5]}$ However, the bandgap opened is usually small unless the nanoribbons are very narrow, and room-temperature electrical applications typically require materials with bandgaps of larger than $0.4 \mathrm{eV}$ to achieve a satisfactory operating on/off ratio. ${ }^{[6]}$ Alternatively, introducing regularly-spaced pores (known as antidot lattices) in graphene can open a reasonable energy bandgap of the order of $1 \mathrm{eV}$ or more. First-principles calculations have predicted that semimetallic graphene can be tailored into a semiconductor by introducing 
an antidot lattice into the graphene sheet. ${ }^{[7]}$ In addition, the energy gap of graphene can be modulated by changing the geometry, size and edges of the pores. Experimentally, the formation of nanoporous graphene (or graphene nanomesh) was first realized by top-down lithography. ${ }^{[8]}$ However, the top-down large scale fabrication of graphene sheets with uniform nano-size pores is challenging.

The bottom-up strategy has shown many advantages for synthesizing atomically precise carbon nanostructures. ${ }^{[6,9]}$ This strategy is based on covalently crosslinking of highly reactive molecular building blocks into carbon nanostructures on a metallic surface, typically via Ullman reactions. ${ }^{[10]}$ Through rational molecular design, on-surface synthesis has demonstrated the formation of well-ordered low-dimensional carbon nanostructures ranging from nanographene, ${ }^{[11-13]}$ graphene nanoribbons, ${ }^{[14-17]}$ to nanoporous graphene. ${ }^{[18-20]}$ In particular, the on-surface synthesis of nanoporous graphene (porous graphene network) has attracted much research interest due to their controllable porous structures and electronic properties with suitable bandgaps. ${ }^{[21-23]}$ To date, the large-scale on-surface synthesis of longrange ordered nanoporous graphene remains a major challenge. ${ }^{[24]}$ Detailed studies of the reaction mechanisms and electronic properties of nanoporous graphene are needed to realize the ultimate goal of commercial applications of the bottom-up nanoporous graphene. Moreno et al. has demonstrated the bottom-up synthesis of nanoporous graphene via the careful control of three thermally-induced reaction steps, namely the Ullmann reaction to polymerize the precursor molecule, cyclodehydrogenation to create 1D graphene nanoribbons, followed by the cross-coupling of graphene nanoribbons into 2D nanoporous graphene with $1 \mathrm{~nm}$ pores. Scanning tunneling spectroscopy reveals that this as-synthesized nanoporous graphene is a semiconductor with a band gap of $\sim 1 \mathrm{eV} \cdot{ }^{[25]}$

In this work, we demonstrate the on-surface synthesis of atomically-thin nanoporous graphene via a simple two-step polymerization using a bromine-terminated polycyclic 
aromatic hydrocarbon molecule precursor (Hexakis(4-bromophenyl)benzene). Two different types of nanoporous graphene (labeled as nanoporous graphene-1 and nanoporous graphene2) were synthesized via the hierarchical dehalogenation coupling and subsequent dehydrogenation reaction, respectively. The reaction processes and atomic structures of nanoporous graphene were probed in-situ by low-temperature scanning tunneling microscopy (LT-STM) and non-contact atomic force microscopy (nc-AFM) with CO-terminated tip. Scanning tunneling spectroscopy (STS) technique was employed to probe their respective electronic bandgaps. Uniform nanopores about $0.6 \mathrm{~nm}$ in diameter were found in nanoporous graphene-2. The quasiparticle bandgaps of as-synthesized nanoporous graphene-1 and nanoporous graphene- 2 were measured to be $5.0 \pm 0.1 \mathrm{eV}$ and $3.8 \pm 0.1 \mathrm{eV}$, respectively. This demonstrates the effectiveness of this simple thermal method for converting the zero-gap pristine graphene into a moderate-gap semiconductor (nanoporous graphene-2), or into a wide-gap insulator (nanoporous graphene-1). Density-functional theory (DFT) calculations verify the STS measurements, and attribute the bandgap opening in nanoporous graphene- 2 to the local confinement of $\pi$-electrons induced by pore generation. The wide bandgap in nanoporous graphene- 1 is ascribed to the non-planar structure hindering the $\pi$-electron delocalization in the nanostructures.

\section{Results and Discussion}

\subsection{Halogen bond induced formation of the supramolecular structure}

Hexakis(4-bromophenyl)benzene (HBPB, a 3D molecular structure is shown in Figure S1) was synthesized as the molecular precursor for nanoporous graphene. The detailed information for the synthesis of HBPB precursors is provided in Scheme $\mathbf{S 1}$ in the Methods section and Supplementary Materials. The HBPB precursors were thermally evaporated on $\mathrm{Au}(111)$ substrate held at room temperature. Figure 1a shows a large-scale STM image of a single layer of the HBPB on $\mathrm{Au}(111)$. The HBPB molecules form a well-ordered high- 
coverage self-assembled structure. The high-resolution atomic structure of the HBPB molecules is presented in Figure 1b. The herringbone reconstruction of $\mathrm{Au}(111)$ remains visible after molecular deposition, indicating the physisorption nature of HBPB on $\mathrm{Au}(111)$. Each HBPB molecule can be clearly identified, suggesting that the crosslink reaction does not occur upon room-temperature deposition. The six-fold symmetric spokes (labeled by blue dashed circles) are assigned to the peripheral phenyl groups of HBPB, and the fainter peripheral dots (red dashed circles) are assigned to $\mathrm{Br}$ atoms bonded to the phenyl groups. The central dark region is assigned to the central phenyl group (black dashed circle). Figure 1c exhibits the corresponding cross-section profile of a single HBPB molecule (along blue line in Figure 1b). The STM cross-sectional line profile (XY) suggests that the apparent height of the peripheral phenyl groups is estimated to be $35 \mathrm{pm}$ higher than that of the central phenyl group. Although the STM image provides electronic topography, the brighter surrounding features suggest an out-of-plane property of the peripheral phenyl groups. This is in good agreement with the computed energy minimized structure that shows a twist angle of 66 degrees due to steric hindrance (Figure 1d and Figure S1). The STM line profile in Figure 1c shows the two peripheral phenyl groups, and the two lower protrusions correspond to the two $\mathrm{Br}$ atoms at each side of the molecule. Figure 1d shows the molecular packing structure of the supramolecular monolayer after geometric relaxation. The theoretical $\mathrm{Br}-\mathrm{Br}$ distance is $\sim 4.0 \AA$ (Figure S2a), which is comparable to the measured value of $3.8 \pm 0.3 \AA$. We therefore propose the stabilization of the self-assembled HBPB superstructure via triple $\mathrm{Br}-\mathrm{Br}$ halogen bonds labeled by the triangular dashed lines. The $\mathrm{Br}$ atoms at the outmost part of HBPB molecules are negatively-charged, and the anisotropic distribution of their electron density promotes the coexistence of electron-depleted $\delta$-holes at the pole of the $\mathrm{C}-\mathrm{Br}$ bond, and encircling electron-rich equatorial belt perpendicular to the $\mathrm{C}-\mathrm{Br}$ bond (Figure $\mathbf{S 2 b}$ ). ${ }^{[26]}$ The mutual interaction between the $\delta$-hole and the electron-rich belt facilitates the formation of triple Br-Br halogen bonds (see Figure S2). Our finding is consistent with the previous 
report on the formation of triple I-I halogen bonds in the molecular tessellation stabilized by the synergistic effect of halogen-Au coordination and halogen bond. ${ }^{[27]} \mathrm{We}$ did not observe the coexistence of halogen-Au coordination and halogen bond in $\mathrm{HBPB} / \mathrm{Au}(111)$. This may be due to the relatively stronger chemical bonding of $\mathrm{Br}-\mathrm{C}$ bond compared to the $\mathrm{I}-\mathrm{C}$ bond that hinders the formation of $\mathrm{Br}-\mathrm{Au}$ coordination. As a result, the triple $\mathrm{Br}-\mathrm{Br}$ halogen bond promotes HBPB self-assembly into a uniform supramolecular monolayer with a unit cell of $1.48 \mathrm{~nm} \times 1.48 \mathrm{~nm}($ Figure 1b).

\subsection{On-surface synthesis of nanoporous graphene}

Figure 2 displays the on-surface synthesis of nanoporous graphene-1 and nanoporous graphene-2. Figure 2a outlines the reactive pathway of nanoporous graphene-1 and nanoporous graphene-2 via Ullman coupling and dehydrogenation, respectively. The HBPB building blocks are cross-linked to each other via C-C coupling to form nanoporous graphene1. Figure $2 \mathrm{~b}$ shows a STM image of the nanoporous graphene-1 on $\mathrm{Au}(111)$, obtained after the HBPB supramolecular monolayer was heated to $250{ }^{\circ} \mathrm{C}$. Due to desorption of some precursor molecules, low coverage nanoporous graphene-1 formed. The molecular structure of nanoporous graphene-1 is superimposed in a zoom-in STM image in Figure 2c. In this arrangement, a building block consists of six symmetric protrusions and a central dark region. The former can be assigned to six peripheral phenyl groups and the latter to the central phenyl group. The distinctive contrast between the central and peripheral phenyl groups originate from the twisted phenyl groups, as previously discussed. The cross-section profile in Figure S3 suggests that the thickness of nanoporous graphene-1 is about $2.6 \AA$, similar to that of the HBPB precursor monolayer (2.6 $\AA$ in Figure S4). STM gives an electronic image, and this STM height is smaller than the calculated $4.1 \AA$. The intermediates of the unreacted activated precursor are observed after annealing at $160{ }^{\circ} \mathrm{C}$ in Figure S6, and the dissociated $\mathrm{Br}$ atoms can be observed (indicated by blue arrows). The resulting new supramolecular network is likely stabilized by underlying Au substrate atoms. ${ }^{[28-29]}$ The six peripheral protrusions that 
correspond to $\mathrm{Br}$ atoms in Figure 1b disappear after annealing to $250{ }^{\circ} \mathrm{C}$, indicating the dissociation of $\mathrm{Br}$ atoms from the peripheral phenyl group. A head-to-head linkage forms between two adjacent peripheral phenyls, indicating the formation of crosslinked C-C bonds via the Ullman reaction after the dissociation of $\mathrm{Br}$ atoms. A crosslinked site and the two central phenyls are labeled by the red arrow and black arrow, respectively (Figure $2 \mathrm{~b}$ ). In summary, the generation of nanoporous graphene-1 is facilitated through an Ullman reaction pathway of thermally-induced halogen atom dissociation and subsequent $\mathrm{C}-\mathrm{C}$ coupling. The proposed reaction pathway of nanoporous graphene-1 synthesis is outlined in Scheme S2.

Nanoporous graphene-2 was synthesized from nanoporous graphene-1 via dehydrogenation reaction, as outlined in Figure 2a. Figure S5a presents a large-scale STM image of the nanoporous graphene- 2 obtained via annealing the nanoporous graphene- 1 at $380{ }^{\circ} \mathrm{C}$ on $\mathrm{Au}(111)$. Figure $2 \mathrm{~d}$ shows a high-resolution STM image of low-coverage nanoporous graphene-2. The STM image shows that nanoporous graphene-2 has a smoother structure, and the six symmetric protrusions (as observed in Figure 2b) disappear, suggesting the elimination of the twist structure after dehydrogenation reaction. The nominal thickness of nanoporous graphene-2 is $\sim 0.18 \mathrm{~nm}$ (see cross-section profile in Figure $\mathbf{S 5 b}$ ). This is comparable to the typical thickness $(0.15-0.20 \mathrm{~nm})$ of single-atom thin graphene or graphene nanoribbons on $\mathrm{Au}$ substrates, ${ }^{[5,30]}$ suggesting a single-atom thickness of the as-synthesized nanoporous graphene-2. In contrast, due to the non-planar structure of precursor and nanoporous graphene-1, their corresponding thickness (0.26 nm in Figure $\mathbf{S 3}$ and S4) is higher than that of nanoporous graphene-2. We noted a similar planar structure of single-atom thick graphene nanoribbons was also observed after the elimination of the out-of-plane structure in precursors at $348{ }^{\circ} \mathrm{C}{ }^{[15]}$. Thus, a higher temperature treatment here $\left(380{ }^{\circ} \mathrm{C}\right) \mathrm{can}$ also trigger a dehydrogenation reaction following by $\mathrm{C}-\mathrm{C}$ coupling, leading to the formation of the planar nanoporous graphene-2. The proposed top-view and side-view atomic structures are therefore presented in Figure 2a. 
To confirm the proposed atomic structure, we carried out non-contact atomic force microscopy (nc-AFM) measurements with a CO-functionalized tip at $4.5 \mathrm{~K}$. Figure 2e and Figure $2 \mathrm{f}$ display a representative constant-current STM image of nanoporous graphene-2 and its corresponding frequency shift $n c$-AFM image, respectively. The $n c$-AFM image (Figure 2f) shows that three C-C linkages (labeled by a blue arrow) form between the three building blocks after dehalogenation. As a result, a pore with a size of $\sim 0.6 \mathrm{~nm}$ was formed. We note that this pore size is over-estimated since $n c$-AFM is not sensitive to hydrogen atom. ${ }^{[31]}$ The enlarged $n c$-AFM image in Figure 2g highlights a single building block (molecular structure is inserted), and clearly shows a hexa-peri-hexabenzocoronene (HBC) structure with 13 hexagons, verifying the formation of six internal C-C bonds after thermally-induced dehydrogenation. The $n c$-AFM measurements thus support the proposed path for the synthesis of nanoporous graphene-2 via hierarchical dehalogenation and dehydrogenation, respectively. We were unable to obtain $n c$-AFM images of nanoporous graphene-1 due to its nonplanarity. ${ }^{[11]}$

\subsection{Electronic bandgap opening in nanopore graphene}

The successful on-surface synthesis and well-defined structural characterization of nanoporous graphenes enable us to further investigate their structure-correlated electronic properties. Scanning tunneling spectroscopy (STS) was employed to probe the electronic band structure of nanoporous graphenes to resolve the respective valence band (VB) and conduction band (CB) features. Figure 3 displays the characteristic dI/dV spectra of both nanoporous graphene-1 and nanoporous graphene-2. The $\mathrm{dI} / \mathrm{dV}$ spectra of the nanoporous graphene-1 were recorded as indicated at the nanopore edges (typically labeled by red dot in the STM image inset) in Figure 3a. More than 50 STS measurements were carried out, and Figure 3a shows the average spectra. The valence band (VB) is a shoulder peak at $-1.70 \pm$ $0.05 \mathrm{eV}$. The conduction band $(\mathrm{CB})$ is a prominent peak at $3.30 \pm 0.05 \mathrm{eV}$. The $\mathrm{dI} / \mathrm{dV}$ spectra 
of the bare Au substrate (recorded at the blue dot in the STM inset) were presented for tip calibration. Thus, the quasiparticle bandgap of the nanoporous graphene- 1 is $5.0 \pm 0.1 \mathrm{eV}$. We postulate that the large bandgap is due to the twist feature of the phenyl groups (Figure 2b), which prevents orbital hybridization and delocalization of $\pi$-electrons (as supported by DFT calculations in Figure 4). To verify this, we compare the dI/dV spectra on a single HBPB molecule. Figure $\mathbf{S 7}$ shows that the electronic HOMO-LUMO gap of self-assembled HBPB monolayer is $5.02 \pm 0.10 \mathrm{eV}$, suggesting that the energy gap did not differ significantly in nanoporous graphene-1 compared to the HBPB monolayer.

In contrast, nanoporous graphene-2 exhibits semiconducting properties as shown in Figure 3b. The STS acquisition points were taken at the red dot in the inserted STM image. The valence band (VB) can be determined as the shoulder at $-1.54 \pm 0.05 \mathrm{eV}$. The conduction band $(\mathrm{CB})$ is the prominent peak at $2.31 \pm 0.04 \mathrm{eV}$. As a result, a quasiparticle bandgap of $3.85 \pm 0.05 \mathrm{eV}$ was extracted, suggesting the semiconducting nature of the as-synthesized nanoporous graphene- 2 .

To compare and verify the as-synthesized pore graphene with other semimetallic and insulating 2D materials, we also carried out STS measurements of highly oriented pyrolytic graphite (HOPG) and few-layered h-BN. Figure S8 shows the STM images and STS spectrum of HOPG. The STM image with a lattice constant of $0.24 \mathrm{~nm}$ shows that the HOPG consists of A-B stacking graphene due to the trigonal symmetry of the bright dots. ${ }^{[32]}$ The STS spectra show a sharp "V" shape near the Fermi level, suggesting semi-metallic zero bandgap behavior in graphite, as expected. Nanoporous graphene-2 refers to a graphene with nanopore. However, compared with zero-gapped graphene, nanoporous graphene-2 has a wide bandgap of $3.85 \mathrm{eV}$. Thus, the nanopore structure turns semimetallic graphene into a semiconductor.

Figure S9 shows STM images and STS spectra of few-layered h-BN. The STM images show that h-BN has a lattice constant of $0.24 \mathrm{~nm}$, similar to that of graphene. However, the 
STS spectra of few-layered h-BN display a wide " $U$ " shape with a bandgap of $5.70 \mathrm{eV}$, suggesting an insulating property. This result is in good agreement with previous experiment and calculation reports. ${ }^{[33]}$ We note that as-synthesized nanoporous graphene-1 has a large bandgap comparable to that of insulating h-BN. Nevertheless, the large bandgap opening in nanoporous graphene-1 results from the structural twist of the internal phenyl groups, as discussed later in Figure 4.

\subsection{Calculated structure-correlated electronic properties}

First-principles calculations were performed to complement the previous discussions on the electronic properties of nanoporous graphene. Figure $\mathbf{4 a - 4 c}$ show the top-view and the sideview of atomic structures of a single HBPB monomer, nanoporous graphene-1, and nanoporous graphene-2, respectively. Upon geometric optimization, a twist angle of 66 degrees between internal phenyl groups was observed in both HBPB monomer and nanoporous graphene-1, suggesting non-planar structures in both cases. The calculated layer height for nanoporous graphene- 1 is $\sim 0.41 \mathrm{~nm}$. In contrast, planar nanoporous graphene- 2 is energetically favorable. These calculated geometric structures are consistent with our STM and $n c$-AFM observations.

Figure 4d-4f show the calculated projected density of states (PDOSs) of a single HBPB monomer, nanoporous graphene-1, and nanoporous graphene-2, respectively. The PBE gaps are estimated to be $3.11 \mathrm{eV}, 2.93 \mathrm{eV}$, and $1.97 \mathrm{eV}$ for HBPB molecule, nanoporous graphene1, and nanoporous graphene-2, respectively. We note that the calculated band gaps are smaller than those measured by STS due to the band gap underestimation via PBE exchangecorrelation functionals. ${ }^{[34]}$ The calculation results are consistent with STS measurements in terms of the decreasing trend of the energy gaps. The energy gaps show an insignificant change between single HBPB monomer and nanoporous graphene-1, both of which exhibit a non-planar structure. In contrast, the energy gap of the planar nanoporous graphene- 2 is 
$0.96 \mathrm{eV}$ smaller than those of the non-planar structures. A more delocalized $\pi$-electron in $p_{z^{-}}$ orbital narrows the energy gap of $s p^{2}$-hybridized carbon materials. For example, the HOMOLUMO gaps decrease in extended acenes or size-expanded nanographene. ${ }^{[35-37]}$ Both STS and DFT results exhibited insignificant variation in terms of energy gap of the HBPB precursor and nanoporous graphene-1, indicating that the two cases have a similar $s p^{2}$-hybridized state. We propose that the $s p^{2}$-hybridized states remain unchanged due to their twisted feature of internal phenyl groups that eventually limits the $\pi$-electron delocalization ( $s p^{2}$-hybridized). After eliminating the twisted feature via subsequent dehydrogenation reaction, a substantial bandgap narrowing was observed in planar nanoporous graphene-2. We further confirmed the effect of twisted phenyl groups on both the $s p^{2}$-hybridized states and the energy gap by considering their respective $p_{\mathrm{z}^{-}}, p_{\mathrm{x}^{-}}$, and $p_{\mathrm{y}^{-}}$PDOS profiles as shown in Figure $4 \mathrm{~d}-\mathrm{f}$. Each $p_{\mathrm{z}^{-}}$, $p_{\mathrm{x}^{-}}$, and $p_{\mathrm{y}^{-}}$orbital contributes equally to the HOMO (or valence band) and LUMO (or conduction band) states in HBPB molecule and nanoporous graphene-1. In contrast, the $p_{z}$ orbital dominates the valence band and conduction band edges in nanoporous graphene-2. It is clear that the twisting of the phenyl groups plays an important role on the dehybridization of the $p_{\mathrm{z}}, p_{\mathrm{x}}$, and $p_{\mathrm{y}}$ orbitals. The planar structure enhances the hybridization of $p_{\mathrm{z}}$-orbital, and thus narrows the energy gap in nanoporous graphene- 2 .

The visualized partial charge density further confirms that the planar structure of nanoporous graphene creates a more delocalized charge density distribution. As shown in Figure 5a-5d, the charge density near valence and conduction band edge is mainly localized at the phenyl groups in nanoporous graphene-1, while the charge density of the planar nanoporous graphene- 2 is more delocalized and extended through the whole carbon network. These results are consistent with the STM observations in Figure 2 b and Figure 2 d. Therefore, a good agreement between partial charge density distribution and STM images 
suggests that the planar structure in nanoporous graphene-2 (compared with nanoporous graphene-1) promotes charge density delocalization and results in a reduced bandgap.

The large bandgap opening in nanoporous graphene-2 is consistent with Clar sextet theory. Clar sextet theory has been proposed to explain the electronic structure in polycyclic aromatic hydrocarbon systems, graphene nanoribbons, and carbon nanotubes. ${ }^{\left[{ }^{[3-39]}\right.}$ It has also been used to explain the energy bandgap opening in nanoporous graphene (or graphene antidot lattices). ${ }^{[40]}$ Clar's aromatic $\pi$-sextets can be defined as the formation of localized six $\pi$ electrons that are localized within a single phenyl ring separated from the adjacent hexagons by $\mathrm{C}-\mathrm{C}$ single bonds. ${ }^{[41]}$ According to Clar sextet theory, a planar nanographene could have a larger bandgap if it has a higher ratio between the numbers of Clar sextets and the total number of hexagons in the unit cell. ${ }^{[38]}$ Clar structure analysis was carried out to obtain Clar sextets distribution in nanoporous graphene-2 (Figure 5e) and Moreno's nanoporous graphene (Figure 5f). The Clar sextet ratio of nanoporous graphene-2 is 7/13 (Figure 5e). This high ratio of Clar sextet opens up a large bandgap of $\sim 3.8 \mathrm{eV}$ in nanoporous graphene- 2 . In contrast, the Clar sextet ratio of Moreno's nanoporous graphene is 6/14 in Figure $5 f$. In addition, a structural resonance forms in Moreno's nanoporous graphene, suggesting the efficient $\pi$-electron delocalization in Moreno's conjugated carbon structure. The smaller Clar sextet ratio and the delocalized $\pi$-electron of Moreno's conjugated nanoporous graphene results in a narrow bandgap of $\sim 1.0 \mathrm{eV} .{ }^{[25]}$ While the higher ratio of Clar sextets and the nonresonance aromatic structure suppresses the $\pi$-electrons delocalization and thereby promotes a large bandgap opening in nanoporous graphene-2.

\section{Conclusion}

Low-dimensional carbon-based materials with semiconducting properties have attracted much interest since they are promising as a transport component for electronic applications. Mugarza's group demonstrated the on-surface synthesis of nanopous graphene with a narrow 
bandgap of $\sim 1.0 \mathrm{eV}$ by introducing nanopores into the graphene lattice. ${ }^{[25]}$ Here, we report a simple route to synthesize two kinds of nanoporous graphene networks with large bandgaps. Nanoporous graphene-1 and nanoporous graphene-2 were obtained via hierarchical thermallyinduced dehalogenation coupling and dehydrogenation reaction, respectively. The quasiparticle bandgaps of the as-synthesized nanoporous graphene-1 and nanoporous graphene- 2 were determined to be $\sim 5.0 \mathrm{eV}$ and $\sim 3.8 \mathrm{eV}$, respectively. Corroborated by the DFT calculations, we suggest that the large bandgap opening in nanoporous graphene-1 is governed by its non-planar structure that inhibits the $\pi$-electron hybridization throughout the structure. The formation of nanoscale pores leads to a bandgap opening in nanoporous graphene-2, which is consistent with Clar sextet theory. This work demonstrates that zerogapped pristine graphene can be converted into both a semiconductor (nanoporous graphene-2) and a large-gapped insulator (nanoporous graphene-1) through rational structure design (by the inserted nanopores or a twisted structure). Further work is needed to fabricate uniform large area nanographenes for scaling-up for future device applications. The uniform continuous nanographenes could be realized via a rational design of surface reaction with fewer numbers of C-C coupling and higher precursor surface diffusion coefficients.

\section{Experimental Section}

Synthesis of hexakis(4-bromophenyl)benzene (HBPB): Precursor HBPB was synthesized according to the reaction path in scheme S1. The detailed information for the synthesis of HBPB precursors is provided in the Supplementary Materials.

On-surface synthesis of nanoporous graphene: The synthesis of nanoporous graphene was carried out under UHV conditions (below $10^{-9} \mathrm{mbar}$ ). The Au were cleaned via repeated cycles of Argon sputtering and subsequent annealing to $800 \mathrm{~K}$. HBPB precursors were thermally deposited from a Knudsen effusion cell onto metal substrates held at room temperature. After deposition, the sample was directly transferred to STM chamber for 
imaging, or annealed at a controlled temperature then transferred to LT-STM $/ n c-\mathrm{AFM}$ chamber for imaging.

Characterization: LT-STM measurements were carried out in Omicron low-temperature STM interfaced with a Nanonis controller at National University of Singapore. The STM images were obtained in constant current mode using chemically-etched tungsten tips. For $\mathrm{dI} / \mathrm{dV}$ measurement, the tunneling current was collected by a lock-in amplifier with modulation of $675 \mathrm{~Hz}$ and $40 \mathrm{mV}$, and $\mathrm{dI} / \mathrm{dV}$ spectra were also recorded at bare $\mathrm{Au}(111)$ for calibration. The STM measurements were performed at $77 \mathrm{~K}$ under a base pressure better than $10^{-10}$ mbar, while $n c$-AFM at $4.5 \mathrm{~K}$ with a base pressure of $10^{-11}$ mbar.

Computational Details: For the calculation details, please refer to section S2.

\section{Supporting Information}

Supporting Information is available from the Wiley Online Library or from the author.

\section{Acknowledgements}

D. W. and X. L. contributed equally to this work. A.T.S. W. and J. W. supervised the project.

D. W. performed LT-STM and nc-AFM measurements. D. W., M. Y., A.T.S. W., J. W. and A. analyzed and discussed the data. M. Y. contributed to DFT calculations. X. L. synthesized the organic precursor under the supervision of J. W. All authors contributed to the scientific discussion and helped in writing the manuscript. We acknowledge financial support from the MOE Tier 3 program (MOE2014-T3-1-004), MOE Tier 2 project (MOE2018-T2-1-088). We also acknowledge Dr. Su Jie and Dr. Gou Jian for technical support. We would like to thank the Centre for Advanced 2D materials and Center of Information Technology at NUS for the computational resource. 
Received: ((will be filled in by the editorial staff))

Revised: ((will be filled in by the editorial staff))

Published online: ((will be filled in by the editorial staff))

\section{References}

[1] K. S. Novoselov; V. I. Fal'ko; L. Colombo; P. R. Gellert; M. G. Schwab; K. Kim, Nature 2012, 490, 192-200.

[2] X. Xu; C. Liu; Z. Sun; T. Cao; Z. Zhang; E. Wang; Z. Liu; K. Liu, Chem. Soc. Rev. 2018, 47, 3059-3099.

[3] F. Schwierz, Nat. Nanotech. 2010, 5, 487-496.

[4] Y. Zhang; T.-T. Tang; C. Girit; Z. Hao; M. C. Martin; A. Zettl; M. F. Crommie; Y. R. Shen; F. Wang, Nature 2009, 459, 820-823.

[5] J. Cai; P. Ruffieux; R. Jaafar; M. Bieri; T. Braun; S. Blankenburg; M. Muoth; A. P. Seitsonen; M. Saleh; X. Feng; K. Müllen; R. Fasel, Nature 2010, 466, 470-473.

[6] L. Talirz; P. Ruffieux; R. Fasel, Adv. Mater. 2016, 28, 6222-6231.

[7] T. G. Pedersen; C. Flindt; J. Pedersen; N. A. Mortensen; A.-P. Jauho; K. Pedersen, Phys. Rev. Lett. 2008, 100, 136804.

[8] J. Bai; X. Zhong; S. Jiang; Y. Huang; X. Duan, Nat. Nanotech. 2010, 5, 190-194.

[9] Q. Sun; R. Zhang; J. Qiu; R. Liu; W. Xu, Adv. Mater. 2018, 30, 1705630.

[10] L. Grill; M. Dyer; L. Lafferentz; M. Persson; M. V. Peters; S. Hecht, Nat. Nanotech. 2007, 2, 687-691.

[11] K. Xu; J. I. Urgel; K. Eimre; M. Di Giovannantonio; A. Keerthi; H. Komber; S. Wang; A. Narita; R. Berger; P. Ruffieux; C. A. Pignedoli; J. Liu; K. Müllen; R. Fasel; X. Feng, J. Am. Chem. Soc. 2019, 141, 7726-7730.

[12] J. Su; M. Telychko; P. Hu; G. Macam; P. Mutombo; H. Zhang; Y. Bao; F. Cheng; Z.-Q. Huang; Z. Qiu; S. J. R. Tan; H. Lin; P. Jelínek; F.-C. Chuang; J. Wu; J. Lu, Sci. Adv. 2019, 5 , eaav7717.

[13] B. Yang; B. Dong; L. Chi, ACS Nano 2020, 14, 6376-6382.

[14] D. J. Rizzo; G. Veber; T. Cao; C. Bronner; T. Chen; F. Zhao; H. Rodriguez; S. G. Louie; M. F. Crommie; F. R. Fischer, Nature 2018, 560, 204-208.

[15] P. Ruffieux; S. Wang; B. Yang; C. Sánchez-Sánchez; J. Liu; T. Dienel; L. Talirz; P. Shinde; C. A. Pignedoli; D. Passerone; T. Dumslaff; X. Feng; K. Müllen; R. Fasel, Nature 2016, 531, 489-492.

[16] M. Ammon; T. Sander; S. Maier, J. Am. Chem. Soc. 2017, 139, 12976-12984.

[17] B. Cirera; A. Sánchez-Grande; B. de la Torre; J. Santos; S. Edalatmanesh; E. RodríguezSánchez; K. Lauwaet; B. Mallada; R. Zbořil; R. Miranda; O. Gröning; P. Jelínek; N. Martín; D. Ecija, Nat. Nanotech. 2020, 15, 437-443.

[18] Q. Fan; J. M. Gottfried; J. Zhu, Acc. Chem. Res. 2015, 48, 2484-2494.

[19] Q. Li; B. Yang; J. Björk; Q. Zhong; H. Ju; J. Zhang; N. Cao; Z. Shi; H. Zhang; D. Ebeling; A. Schirmeisen; J. Zhu; L. Chi, J. Am. Chem. Soc. 2018, 140, 6076-6082.

[20] C. Steiner; J. Gebhardt; M. Ammon; Z. Yang; A. Heidenreich; N. Hammer; A. Görling; M. Kivala; S. Maier, Nat. Commun. 2017, 8, 14765.

[21] G. Galeotti; F. De Marchi; E. Hamzehpoor; O. MacLean; M. Rajeswara Rao; Y. Chen; L. V. Besteiro; D. Dettmann; L. Ferrari; F. Frezza; P. M. Sheverdyaeva; R. Liu; A. K. Kundu; P. Moras; M. Ebrahimi; M. C. Gallagher; F. Rosei; D. F. Perepichka; G. Contini, Nat. Mater. 2020, 19, 874-880. 
[22] D. Wang; Z. Wang; W. Liu; Arramel; J. Zhou; Y. P. Feng; K. P. Loh; J. Wu; A. T. S. Wee, ACS Nano 2020, 14, 14008-14016.

[23] P. H. Jacobse; R. D. McCurdy; J. Jiang; D. J. Rizzo; G. Veber; P. Butler; R. Zuzak; S. G. Louie; F. R. Fischer; M. F. Crommie, J. Am. Chem. Soc. 2020, 142, 13507-13514.

[24] W. Liu; X. Luo; Y. Bao; Y. P. Liu; G.-H. Ning; I. Abdelwahab; L. Li; C. T. Nai; Z. G. Hu; D. Zhao; B. Liu; S. Y. Quek; K. P. Loh, Nat. Chem. 2017, 9, 563-570.

[25] C. Moreno; M. Vilas-Varela; B. Kretz; A. Garcia-Lekue; M. V. Costache; M. Paradinas; M. Panighel; G. Ceballos; S. O. Valenzuela; D. Peña; A. Mugarza, Science 2018, 360, 199-203.

[26] Z. Han; G. Czap; C.-1. Chiang; C. Xu; P. J. Wagner; X. Wei; Y. Zhang; R. Wu; W. Ho, Science 2017, 358, 206-210.

[27] F. Cheng; X.-J. Wu; Z. Hu; X. Lu; Z. Ding; Y. Shao; H. Xu; W. Ji; J. Wu; K. P. Loh, Nat. Commun. 2018, 9, 4871.

[28] S. Mishra; D. Beyer; K. Eimre; S. Kezilebieke; R. Berger; O. Gröning; C. A. Pignedoli; K. Müllen; P. Liljeroth; P. Ruffieux; X. Feng; R. Fasel, Nat. Nanotech. 2020, 15, 22-28.

[29] Q. Sun; B. V. Tran; L. Cai; H. Ma; X. Yu; C. Yuan; M. Stöhr; W. Xu, Angew. Chem. Int. Ed. 2017, 56, 12165-12169.

[30] P. Leicht; L. Zielke; S. Bouvron; R. Moroni; E. Voloshina; L. Hammerschmidt; Y. S. Dedkov; M. Fonin, ACS Nano 2014, 8, 3735-3742.

[31] L. Gross; F. Mohn; N. Moll; P. Liljeroth; G. Meyer, Science 2009, 325, 1110-1114.

[32] D. A. Kroeger; E. Cisternas; J. D. Correa, Surf. Sci. 2015, 634, 31-36.

[33] D. Liu; X. Chen; Y. Yan; Z. Zhang; Z. Jin; K. Yi; C. Zhang; Y. Zheng; Y. Wang; J. Yang; X. Xu; J. Chen; Y. Lu; D. Wei; A. T. S. Wee; D. Wei, Nat. Commun. 2019, 10, 1188.

[34] T. Joshi; C. Chen; H. Li; C. S. Diercks; G. Wang; P. J. Waller; H. Li; J.-L. Bredas; O. M. Yaghi; M. F. Crommie, Adv. Mater. 2019, 31, 1805941.

[35] J. Krüger; F. Eisenhut; D. Skidin; T. Lehmann; D. A. Ryndyk; G. Cuniberti; F. García; J. M. Alonso; E. Guitián; D. Pérez; D. Peña; G. Trinquier; J.-P. Malrieu; F. Moresco; C. Joachim, ACS Nano 2018, 12, 8506-8511.

[36] J. Wu; W. Pisula; K. Müllen, Chem. Rev. 2007, 107, 718-747.

[37] X.-Y. Wang; X. Yao; K. Müllen, Sci. China Chem. 2019, 62, 1099-1144.

[38] M. Baldoni; A. Sgamellotti; F. Mercuri, Chem. Phys. Lett. 2008, 464, 202-207.

[39] M. Baldoni; A. Sgamellotti; F. Mercuri, Org. Lett. 2007, 9, 4267-4270.

[40] R. Petersen; T. G. Pedersen; A.-P. Jauho, ACS Nano 2011, 5, 523-529.

[41] M. Solà, Front. Chem. 2013, 1. 

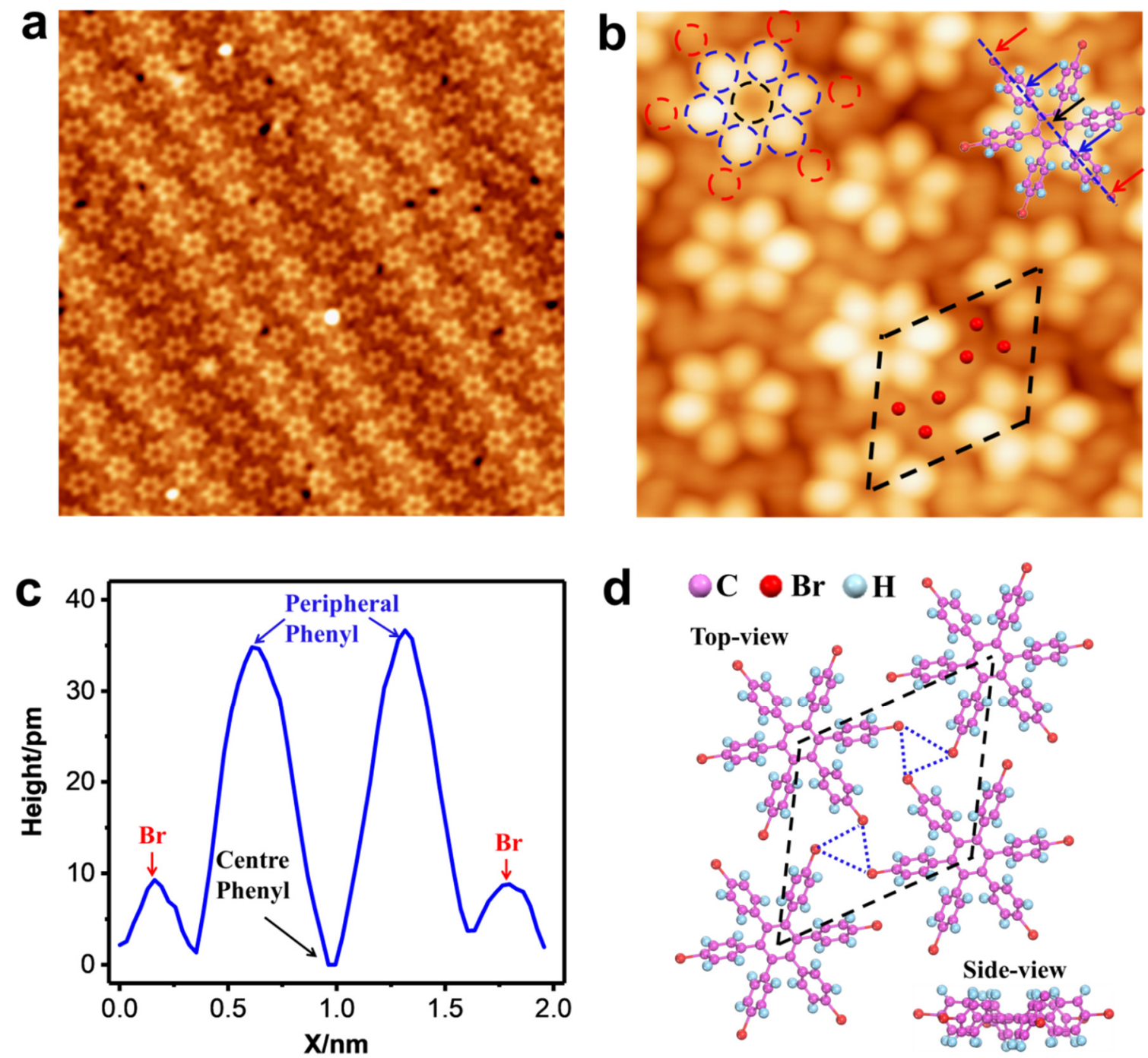

Figure 1. Self-assembly monolayer of HBPB on Au(111) stabilized by triple Br-Br halogen bonds. (a) Large-area STM image of a self-assembly monolayer of roomtemperature deposition of HBPB precursor on $\mathrm{Au}(111)$, set-point: $\mathrm{V}=-0.6 \mathrm{~V}$ and $\mathrm{I}=50 \mathrm{pA}$, size: $20 \mathrm{~nm} \times 20 \mathrm{~nm}$. (b) Atomic-resolution STM image of HBPB precursor, set-point: $\mathrm{V}=$ $0.6 \mathrm{~V}$ and $\mathrm{I}=50 \mathrm{pA}$, size: $4.5 \mathrm{~nm} \times 4.5 \mathrm{~nm}$, a molecular structure of HBPB were superimposed onto the STM image, the inserted black parallelogram delineates a $1.48 \mathrm{~nm} \times$ $1.48 \mathrm{~nm}$ unit cell, central phenyl groups: black dashed circle, peripheral phenyl groups: blue dashed circle, $\mathrm{Br}$ atoms: red dashed circle (or red dots). (c) Corresponding cross-section profile for the blue line in b. (d) A packing configuration of 4 HBPB molecules, side-view of a HBPB molecule is inserted, and triple $\mathrm{Br}-\mathrm{Br}$ halogen bonds are labeled by the triangular blue dashed lines. 

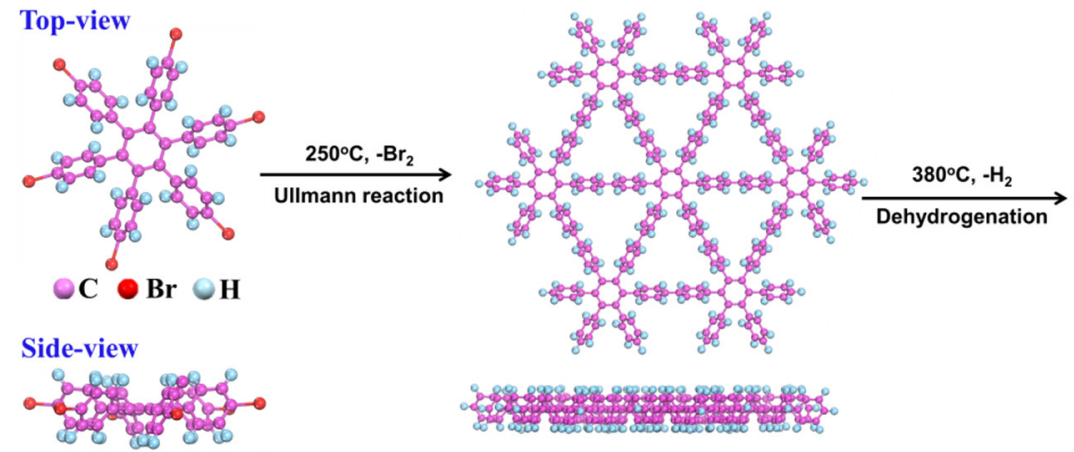

b

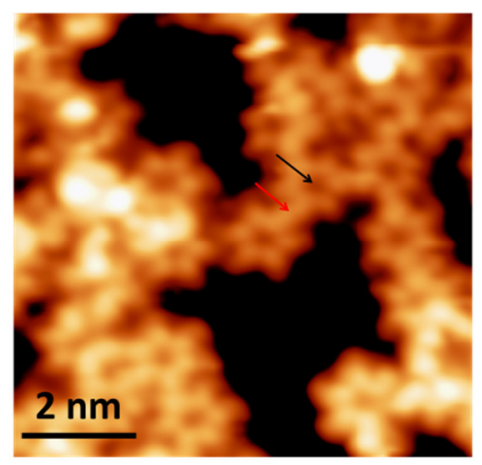

C

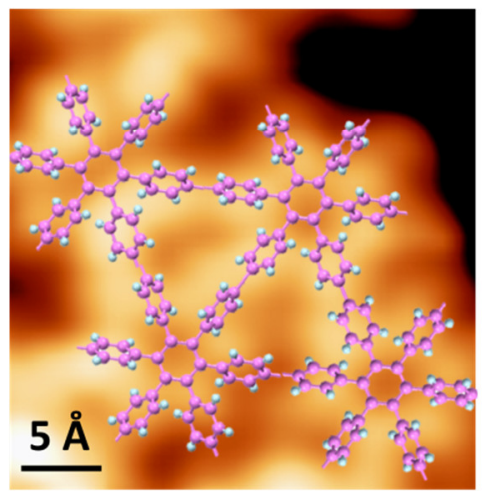

d

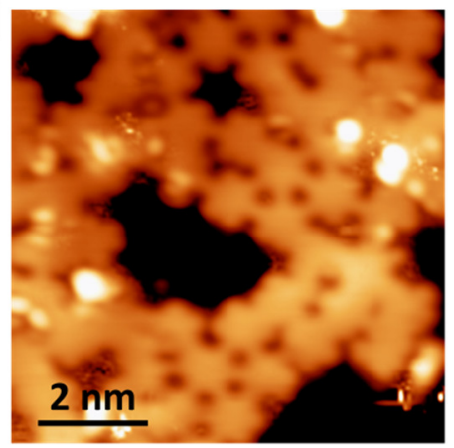

e 0.31

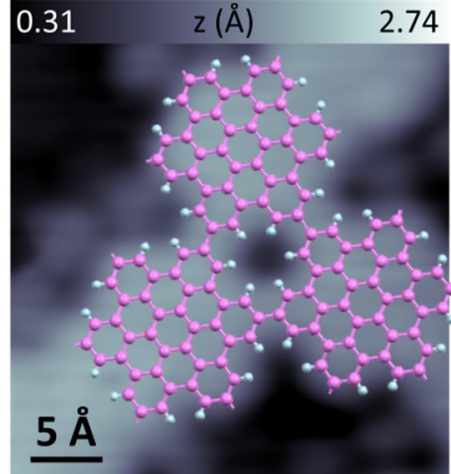

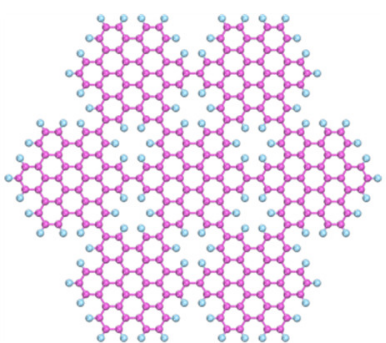

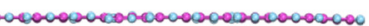
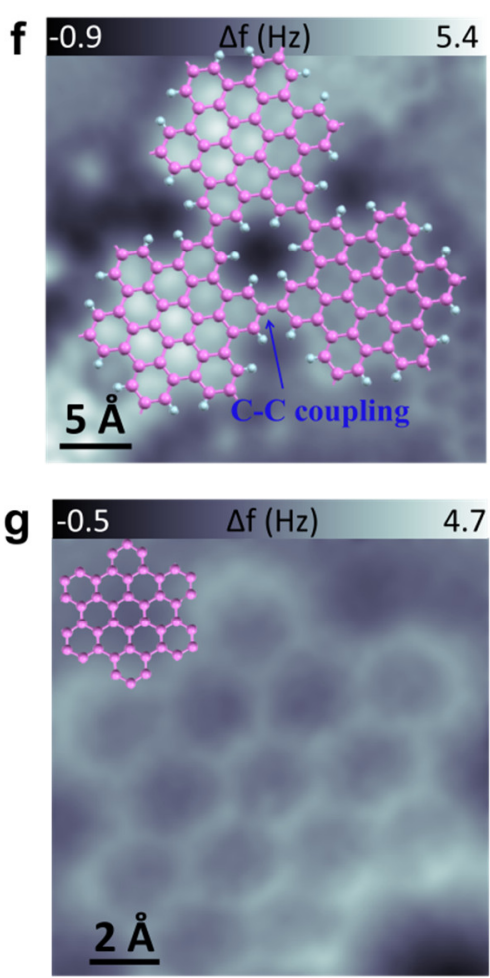

Figure 2. On-surface synthesis of nanoporous graphene-1 and nanoporous graphene-2.

(a) An illustration of surface-assisted synthesis of nanoporous graphene-1 and nanoporous graphene-2 via Ullman coupling and dehydrogenation, respectively; (b) STM image and (c) zoom-in STM image of nanoporous graphene- 1 at $250{ }^{\circ} \mathrm{C}$ on $\mathrm{Au}(111)$, the red arrow and black arrows in (b) label the crosslinked site and central benzenes, respectively; set-point: b) $\mathrm{V}=1.0 \mathrm{~V}$ and $\mathrm{I}=58 \mathrm{pA}, \mathrm{c}) \mathrm{V}=1.0 \mathrm{~V}$ and $\mathrm{I}=58 \mathrm{pA}$; (d) STM image and (e) zoom-in STM image of nanoporous graphene-2 obtained via annealing the nanoporous graphene- 1 at $380{ }^{\circ} \mathrm{C}$, set point: d) $\mathrm{V}=0.5 \mathrm{~V}$ and $\mathrm{I}=40 \mathrm{pA}, \mathrm{e}) \mathrm{V}=30 \mathrm{mV}$ and $\mathrm{I}=21 \mathrm{pA}$; (f, g) Corresponding constant-height $n c$-AFM images of nanoporous graphene-2, obtained with a COfunctionalized tip at $4.5 \mathrm{~K}$, set point: tip height $\mathrm{z}=0 \AA$ with respect to the STM set point in (e), $\mathrm{V}=0 \mathrm{mV}$ and oscillation amplitude $\mathrm{A}=60 \mathrm{pm}$. A structure model of a hexa-perihexabenzocoronene is inserted in $(\mathrm{g})$. A predicted structure model of nanoporous graphene-1 or nanoporous graphene- 2 is superimposed in (c, e and f). 

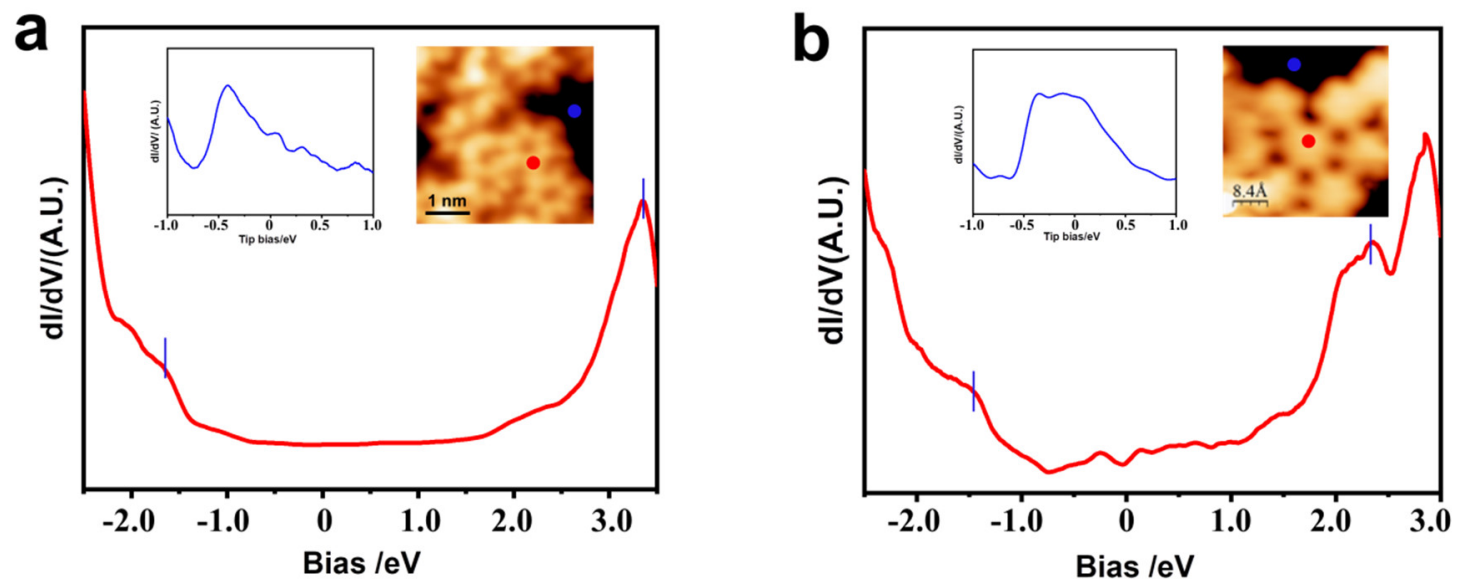

Figure 3. Large bandgaps opens in the as-synthesized nanoporous graphene. (a) Scanning tunneling spectroscopy of nanoporous graphene-1, set-point: $\mathrm{V}=1.0 \mathrm{~V}$ and $\mathrm{I}=60$ $\mathrm{pA}$, the inserted figure is a STM image of nanoporous graphene-1, STS spectra were recorded at red dot, its bandgap was identified as $5.0 \pm 0.1 \mathrm{eV}$; (b) Scanning tunneling spectroscopy of nanoporous graphene-2, set-point: $\mathrm{V}=1.0 \mathrm{~V}$ and $\mathrm{I}=40 \mathrm{pA}$, the inserted figure gives a STM image of nanoporous graphene-2, STS spectra were recorded at red dot, its bandgap was extracted as $3.85 \pm 0.1 \mathrm{eV}$. The inserted $\mathrm{dI} / \mathrm{dV}$ spectra of the bare Au substrate were also recorded at blue dots for tip calibration.

a
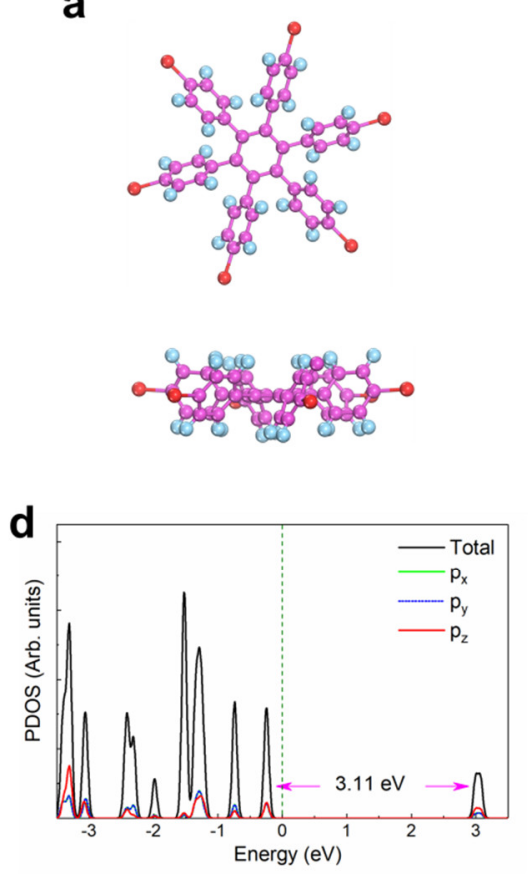

b

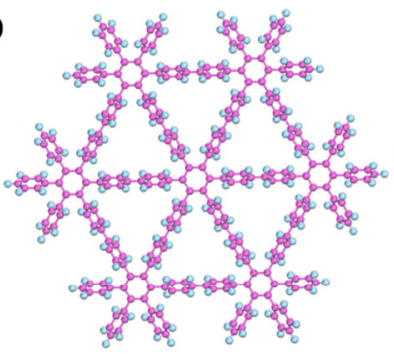

\%8:

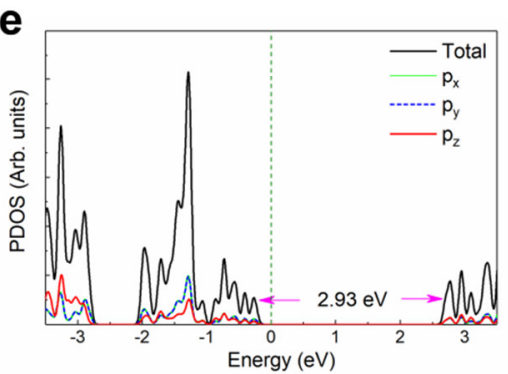

C

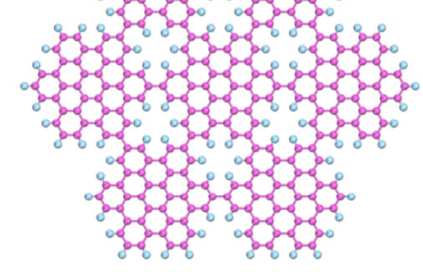

0000000000000000000000000000

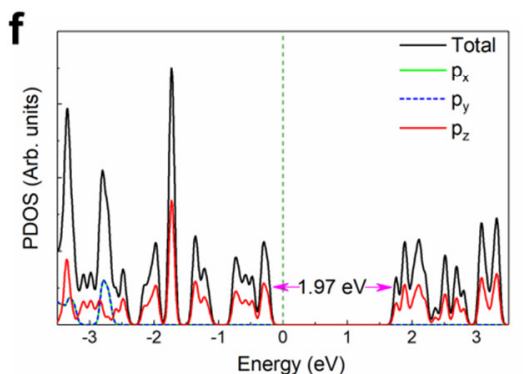

Figure 4. Theoretical electronic properties of the three structures. Optimized atomic structure of (a) a single HBPB monomer, (b) nanoporous graphene-1, and (c) nanoporous graphene-2. Up: Top-view, Bottom: Side-view, in which red, purple and light blue solid balls 
denote $\mathrm{Br}, \mathrm{C}$ and $\mathrm{H}$ atoms, respectively. The calculated PDOSs of (d) a single HBPB monomer, (e) nanoporous graphene-1 and (f) nanoporous graphene-2, in which the PBE energy gaps are extracted as $3.11 \mathrm{eV}, 2.93 \mathrm{eV}, 1.97 \mathrm{eV}$, respectively.

a

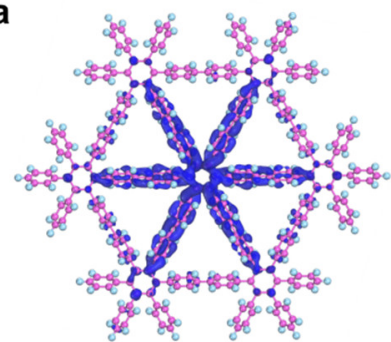

b

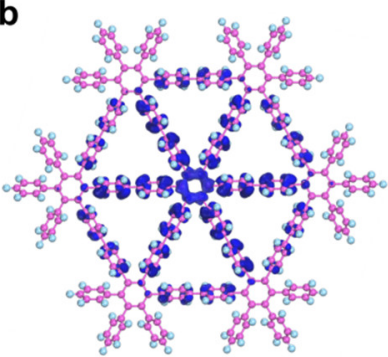

C

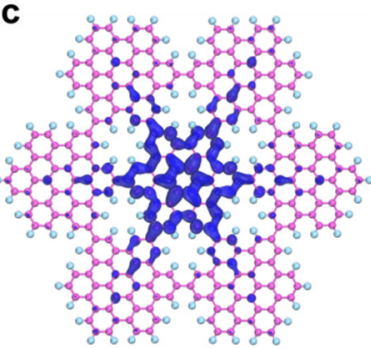

d

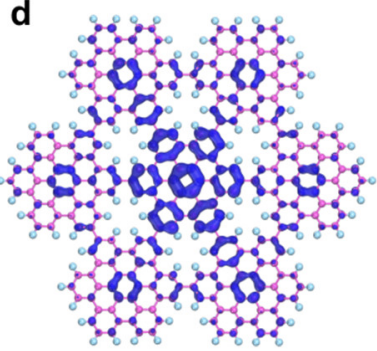

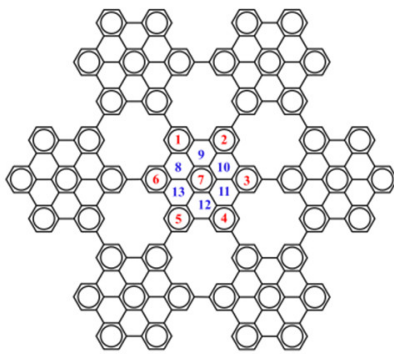

This work

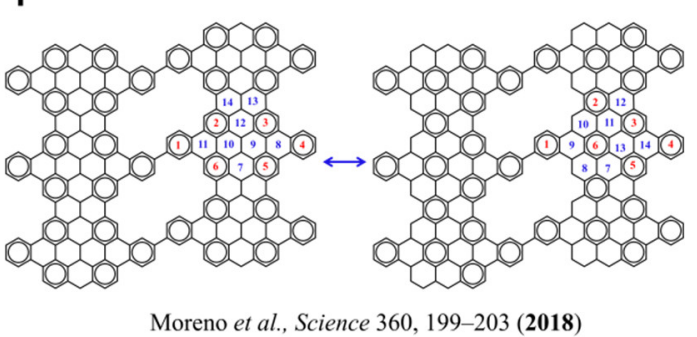

Figure 5. Theoretical partial charge density and Clar structure analysis. The visualized partial charge density of (a, b) nanoporous graphene-1 and (c, d) nanoporous graphene-2 with an iso-surface value of $2.0 \times 1.0^{-3} \mathrm{e} / \AA^{3}$, where $(\mathrm{a}, \mathrm{c})$ and $(\mathrm{b}, \mathrm{d})$ denote partial charge density distribution states near the conduction band and the valence band edge, respectively. Clar structure analysis shows Clar sextets distribution in (e) nanoporous graphene-2 and (f) Moreno's nanoporous graphene. Clar sextet is a delocalized $\pi$-orbital phenyl ring (labeled by central circular rings). As a result, the ratio between the numbers of Clar sextets (labeled by red numbers) and the total number of hexagons in a unit cell is $7 / 13$ (This work) and 6/14 (Moreno's work), respectively. A structural resonance forms in Moreno's nanoporous graphene, suggesting the efficient delocalization of $\pi$-electron. 
Two types of nanoporous graphene were synthesized via a simple way of hierarchical C-C coupling. By introducing nanopores or a twisted structure, semimetallic graphene is converted into semiconducting nanoporous graphene-2 or insulating wide-bandgap nanoporous graphene-1.

D. Wang, X. Lu, Arramel, M. Yang, J. Wu, A. T. S. Wee

\section{Title: On-surface synthesis of variable bandgap nanoporous graphene}

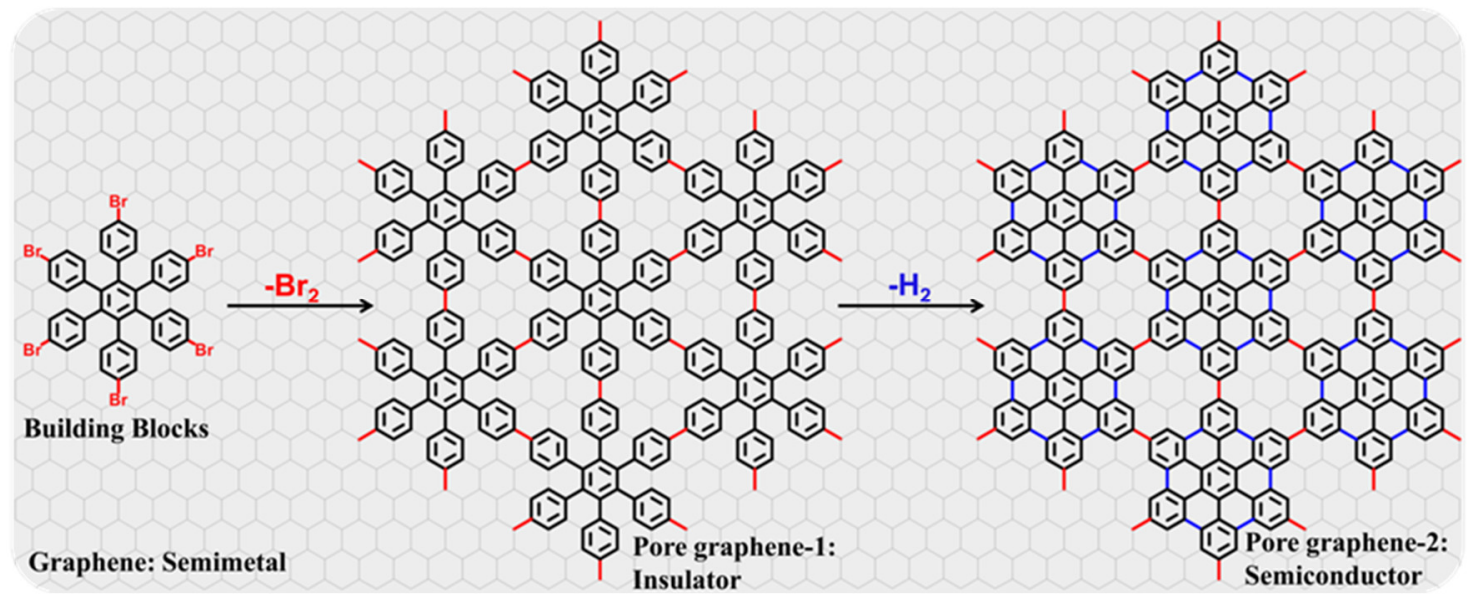

TOC. Surface-assisted bottom-up synthesis of nanoporous graphene with structure and bandgap modulation. 